Pacific

Journal of

Mathematics

A FREE BOUNDARY ISOPERIMETRIC PROBLEM IN HYPERBOLIC 3-SPACE BETWEEN PARALLEL HOROSPHERES

Rosa MARIA BARREIRO CHAVES, Márcio F. DA Silva And Renato H. L. Pedrosa 


\title{
A FREE BOUNDARY ISOPERIMETRIC PROBLEM IN HYPERBOLIC 3-SPACE BETWEEN PARALLEL HOROSPHERES
}

\author{
Rosa MARIA BARREIRo CHAVES, \\ Márcio F. dA Silva ANd Renato H. L. Pedrosa
}

\begin{abstract}
We investigate the isoperimetric problem of finding the regions of prescribed volume with minimal boundary area between two parallel horospheres in hyperbolic 3-space (the part of the boundary contained in the horospheres is not included). We reduce the problem to the study of rotationally invariant regions and obtain the possible isoperimetric solutions by studying the behavior of the profile curves of the rotational surfaces with constant mean curvature in hyperbolic 3-space. We also classify all the connected compact rotational surfaces $M$ of constant mean curvature that are contained in the region between two horospheres, have boundary $\partial M$ either empty or lying on the horospheres, and meet the horospheres perpendicularly along their boundary.
\end{abstract}

\section{Introduction}

Geometric isoperimetric problems, (upper) estimates for the volume of regions of a given fixed boundary volume, and the dual problems play an important role in analysis and geometry. There are both isoperimetric inequalities, common in analysis, and actual classification of optimal geometric objects, like the round ball in Euclidean geometry. We will be interested in the study of a relative free-boundary isoperimetric problem in hyperbolic 3-space between two parallel horospheres. A survey of recent results in isoperimetric problems is [Ritoré and Ros 2002].

For a Riemannian manifold $M^{n}$, we state the classical isoperimetric problem as follows: Classify, up to congruency by the isometry group of $M$, the (compact) regions $\Omega \subseteq M$ enclosing a fixed volume that have minimal boundary volume. The relevant concepts of volume are those of geometric measure theory: Regions and their boundaries are respectively $n$ - and $(n-1)$-rectifiable subsets of $M$; see [Morgan 2009].

MSC2000: 53A10, 49Q10.

Keywords: constant mean curvature surfaces, hyperbolic space, isoperimetric problem.

Da Silva thanks CAPES and CNPq for financial support during his $\mathrm{PhD}$ studies, which led in part to this paper. 
If $M$ has a boundary, the part of $\partial \Omega$ included in the interior of $M$ will be called the free boundary of $\Omega$, and the other part will be called the fixed boundary. One may specify how the fixed boundary of $\Omega$ is included in the computation of the boundary volume functional. In this paper, we will not consider the volume of the fixed boundary of $\Omega$ to be part of the boundary volume functional. We will see in Section 3 that this implies that the angle of contact between the interior boundary of $\Omega$ and $\partial M$ is $\pi / 2$ (when this contact occurs). Such problems are related to the geometry of stable drops in capillarity (the angle of contact depends, as mentioned, on how one considers the volume of the fixed boundary in the computation of the boundary volume functional). For a discussion, see [Finn 1986].

This work is motivated by the well-known results of Athanassenas [1987] and Vogel [1987], which imply that between two parallel planes in Euclidean space $\mathbb{R}^{3}$, a (stable) soap bubble touching both walls perpendicularly is a straight cylinder orthogonal to the planes, and may only exist down to a certain minimal enclosing volume depending on the distance between the planes. Below that value, only half-spheres touching one of the planes or whole spheres not touching either plane occur, and the cylinders become unstable. A new proof of this fact can be found in [Pedrosa and Ritoré 1999], where the authors study the analogous problem in higher-dimensional Euclidean spaces.

In this paper we study the analogous relative isoperimetric problem between two parallel horospheres in the hyperbolic space $\mathbb{H}^{3}(-1)$. We will use the upper half-space model $\mathbb{R}_{+}^{3}$, in which parallel horospheres are represented by horizontal Euclidean 2-planes of $\mathbb{R}_{+}^{3}$. We will classify the possible isoperimetric solutions.

The existence of isoperimetric regions in the manifold with boundary $(B, g)$, the slab composed of the two horospheres and the region between them, may be obtained by adapting a result of Morgan [2009] (applicable since $B / G$ is a compact space, where $G$ is the subgroup of the isometry group of $\mathbb{H}^{3}(-1)$ leaving $B$ invariant). Regarding the regularity of the free boundary, well-known results about the lower codimension bounds of the singular subset imply that it must be regular, and in fact analytic.

In Section 2, we define basic notions in the model $\mathbb{R}_{+}^{3}$, like geodesics, totally geodesic surfaces, umbilical surfaces and rotational surfaces. We also use the area and volume functionals to more precisely formulate the isoperimetric problem.

In Section 3 we get some basic geometric properties of isoperimetric regions; for instance, their (free) boundaries must have constant mean curvature and, when they touch the bounding horospheres, the contact angle must be $\pi / 2$. We also discuss their rotational invariance.

In Section 4 we investigate the tangency of profile curves for the rotational surfaces with constant mean curvature, to determine the possible isoperimetric regions between the two parallel horospheres. We discuss in detail the existence 
of isoperimetric regions and the regularity of the free boundary part, and we prove the following result:

Theorem 1.1. Let $c_{1}, c_{2}$ be positive real constants such that $c_{1}<c_{2}$, and let $\mathscr{F}_{c_{1}, c_{2}}=\left\{(x, y, z) \in \mathbb{R}_{+}^{3}: c_{1} \leq z \leq c_{2}\right\}$. Let $V>0$, and let $\mathscr{C}_{c_{1}, c_{2}, V}$ be the set of $\Omega \subset \mathscr{F}_{c_{1}, c_{2}}$ with volume $|\Omega|=V$ and boundary volume (area) $A\left(\Omega \cap \mathscr{F}_{c_{1}, c_{2}}\right)<\infty$, where we suppose that $\Omega$ is connected, compact and 3-rectifiable in $\mathscr{F}_{c_{1}, c_{2}}$, and has as boundary (between the horospheres) an embedded, orientable, 2-rectifiable surface. Let

$$
A_{c_{1}, c_{2}, V}=\inf \left\{A\left(\Omega \cap \mathscr{\mathscr { F }}_{c_{1}, c_{2}}\right): \Omega \in \mathscr{C}_{c_{1}, c_{2}, V}\right\} .
$$

(1) There exists $\Omega \in \mathscr{C}_{c_{1}, c_{2}, V}$ such that $A\left(\Omega \cap \mathscr{\mathscr { F }}_{c_{1}, c_{2}}\right)=A_{c_{1}, c_{2}, V}$. The free boundaries are analytic surfaces.

(2) If $\Omega$ has minimal boundary volume between the horospheres, the free boundary of $\Omega$ is either

(a) of catenoid cousin type or umbilical with $H=1$,

(b) of equidistant type or umbilical with $0<H<1$, or

(c) of onduloid type or umbilical with $H>1$.

Remark 1.2. We give details of this description in Section 4. The hyperbolic distance $d=\ln \left(c_{2} / c_{1}\right)$ between the horospheres could determine which region among cases (a)-(c) is the isoperimetric solution. It is still not clear, however, which from among (a)-(c) would be solutions for a given $d$. (In [Athanassenas 1987], the classification of isoperimetric solutions depending on $d$ is fully answered for the analogous problem in $\mathbb{R}^{3}$.) In some cases, as in Figure 1 , we know by fixing the lower horosphere at $z=1 / 2$ that umbilical surfaces with $H=1$ cannot be solutions when the upper horosphere is at level $z<1$. In the general case, the question is still open because it is necessary to study the stability of the surfaces (a)-(c) (see [Barbosa et al. 1988] for the notion of stability in this context).

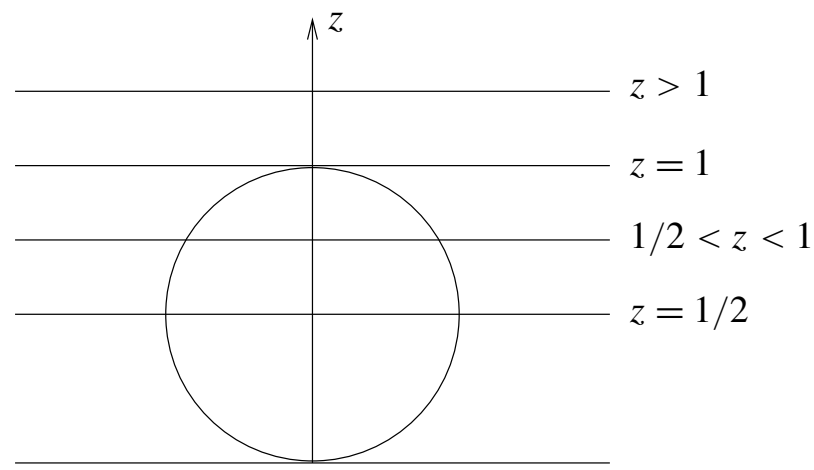

Figure 1. A case in which it is possible to decide. 
Remark 1.3. Theorem 1.1 shows how the situation in hyperbolic geometry differs from that in Euclidean 3-space. In $\mathbb{R}^{3}$, we also have rotationally invariant surfaces of catenoid and onduloid type, but they cannot appear as boundaries of optimizing tubes, even though in higher dimensions, hypersurfaces generated by onduloids in Euclidean space are known to occur as boundaries of optimal tubes connecting two parallel hyperplanes; see [Pedrosa and Ritoré 1999].

Corollary 1.4. Let $M$ be a connected compact rotational surface of constant mean curvature in hyperbolic 3-space. Suppose $M$ is contained in the region between two horospheres, and that the boundary $\partial M$ is either empty or lies on the horospheres, and meets them perpendicularly along its boundary. Then $M$ is either

(1) of catenoid cousin type or umbilical with $H=1$, or

(2) of equidistant type or umbilical with $0<H<1$, or

(3) of onduloid type or umbilical with $H>1$.

\section{Preliminaries}

Let $\mathscr{L}^{4}=\left(\mathbb{R}^{4}, g\right)$ be the 4-dimensional Lorentz space endowed with the metric $g(x, y)=x_{1} y_{1}+x_{2} y_{2}+x_{3} y_{3}-x_{4} y_{4}$ and the 3 -dimensional hyperbolic space

$$
\mathbb{H}^{3}(-1):=\left\{p=\left(x_{1}, x_{2}, x_{3}, x_{4}\right) \in \mathscr{L}^{4}: g(p, p)=-1, x_{4}>0\right\} .
$$

We use the upper half-space model $\mathbb{R}_{+}^{3}:=\left\{(x, y, z) \in \mathbb{R}^{3} ; z>0\right\}$ for $\mathbb{H}^{3}(-1)$, endowed with the metric

$$
\frac{1}{z^{2}}\left(d x^{2}+d y^{2}+d z^{2}\right)
$$

Let $\phi: \Sigma \rightarrow \mathbb{R}_{+}^{3}$ be an isometric immersion of a compact surface $\Sigma$ with nonempty boundary $\partial \Sigma$, and let $\Gamma$ be a curve in $\mathbb{R}_{+}^{3}$. If $\phi$ is a diffeomorphism of $\partial \Sigma$ onto $\Gamma$, we say that $\Gamma$ is the boundary of $\phi$; if $\phi$ has constant mean curvature $H$, we say that $\Sigma$ is an $H$-surface with boundary $\Gamma$. We identify $\Sigma$ with its image by $\phi$ and $\partial \Sigma$ with the curve $\Gamma$.

The plane $z=0$ is called the infinity boundary of $\mathbb{R}_{+}^{3}$ and denoted $\partial_{\infty} \mathbb{R}_{+}^{3}$. The geodesics of $\mathbb{R}_{+}^{3}$ are represented by vertical Euclidean lines and half-circles orthogonal to $\partial_{\infty} \mathbb{R}_{+}^{3}$ and contained in $\mathbb{R}_{+}^{3}$. The totally geodesic surfaces have constant mean curvature $H=0$ and are represented by vertical Euclidean planes and hemispheres orthogonal to $\partial_{\infty} \mathbb{R}_{+}^{3}$ and contained in $\mathbb{R}_{+}^{3}$.

The horizontal Euclidean translations and the rotations around a vertical geodesic are isometries of $\mathbb{R}_{+}^{3}$. We have two families of isometries associated to one point $p_{0} \in \partial_{\infty} \mathbb{R}_{+}^{3}$, the Euclidean homotheties centered at $p_{0}$ with factor $k>0$, called hyperbolic translations through a geodesic $\alpha$ perpendicular to $\partial_{\infty} \mathbb{R}_{+}^{3}$ at $p_{0}$, and the hyperbolic reflections with respect to a totally geodesic surface $P$. 
When $P$ is a hemisphere orthogonal to $\partial_{\infty} \mathbb{R}_{+}^{3}$ centered at $p_{0}$ and of radius $r>0$, the hyperbolic reflections are Euclidean inversions centered at $p_{0}$ that fix $P$. When $P$ is a vertical Euclidean plane, they are Euclidean reflections with respect to $P$.

Now we describe the umbilical surfaces of $\mathbb{R}_{+}^{3}$; see for example [López 1999].

- Totally geodesic surfaces are represented by vertical Euclidean planes in $\mathbb{R}_{+}^{3}$ and the hemispheres in $\mathbb{R}_{+}^{3}$ perpendicular to the plane $z=0$. They have $H=0$.

- Geodesic spheres are represented by Euclidean spheres entirely contained in $\mathbb{R}_{+}^{3}$. They have $H>1$ (the mean curvature vector points to the interior). If $\rho$ is the hyperbolic radius of a geodesic sphere, then $H=\operatorname{coth} \rho$.

- Horospheres are represented by horizontal Euclidean planes in $\mathbb{R}_{+}^{3}$ and Euclidean spheres in $\mathbb{R}_{+}^{3}$ that are tangent to $\partial_{\infty} \mathbb{R}_{+}^{3}$. They have $H=1$; the mean curvature vector points upwards in the case of horizontal planes and to the interior in the case of spheres.

- Equidistant surfaces are represented by the intersection of $\mathbb{R}_{+}^{3}$ with the planes in $\mathbb{R}^{3}$ that are neither parallel nor perpendicular to the plane $z=0$ and by (pieces of) Euclidean spheres that are not entirely contained in $\mathbb{R}_{+}^{3}$ and are neither tangent nor perpendicular to the plane $z=0$. They have $0<H<1$, and the mean curvature vector points to the totally geodesic surface they are equidistant to.

In our study, the (spherical) rotational surfaces of $\mathbb{R}_{+}^{3}$ play an important role since the solutions of the isoperimetric problem must be rotationally invariant. They are defined as surfaces invariant by a subgroup of isometries whose principal orbits are (Euclidean) circles.

Let $\Pi_{1}$ and $\Pi_{2}$ be horospheres represented by distinct parallel horizontal Euclidean planes, and let $\Pi=\Pi_{1} \cup \Pi_{2}$. Let $\mathscr{F}=\mathscr{F}\left(\Pi_{1}, \Pi_{2}\right)$ be the closed slab between them, and let $\phi: \Sigma \rightarrow \mathscr{F}$ be an isometric immersion of a compact, connected, embedded and orientable $C^{2}$ surface with boundary $\Gamma=\partial \Sigma$ and the property that $\phi(\Gamma) \subset \Pi$. (Later we will see that the image under $\phi$ of the interior of the surface $\Sigma$ will not touch $\Pi$ if $\Sigma$ is the boundary of an optimal domain in our variational problem, but this is not part of the general situation yet.)

Now we fix notation for some well-known geometric invariants related to isometric immersions. We (locally) identify $\Sigma$ with $\phi(\Sigma)$ and $X(p) \in T_{p} \Sigma$ with $d \phi_{p}(X(p)) \subset \mathbb{R}_{+}^{3}$. We have the decomposition $T_{p}\left(\mathbb{R}_{+}^{3}\right)=T_{p}(\Sigma) \oplus N_{p}(\Sigma)$ into the tangent and normal spaces to $\Sigma$ at $p$. Choose an orientation for $\Sigma$, and let $N$ be the (positive) unitary normal field along the immersion $\phi$. If $X(p) \in T_{p}\left(\mathbb{R}_{+}^{3}\right)$, we may write $X(p)=X(p)^{T}+X(p)^{N}=X(p)^{T}+\alpha N(p)$, where $\alpha \in \mathbb{R}$.

Let $\langle\cdot, \cdot\rangle$ be the metric induced on $\Sigma$ by the immersion $\phi$, let $\bar{\nabla}$ be the Riemannian connection of the ambient space $\mathbb{R}_{+}^{3}$, and let $\nabla$ be the induced Riemannian 
connection on $\Sigma$. Let $X, Y \in \mathfrak{X}(\Sigma)$ be $C^{\infty}$ vector fields. Then $\nabla_{X} Y=\left(\bar{\nabla}_{X} Y\right)^{T}$ and $\mathscr{B}(X, Y)=\left(\bar{\nabla}_{X} Y\right)^{N}$ are, as usual, the induced connection on $\Sigma$ and the second fundamental form of the immersion given by $\mathscr{B}$. We also have the Weingarten operator $A_{N}(Y)=-\left(\bar{\nabla}_{Y} N\right)^{T}$, so that $\left\langle A_{N}(X), Y\right\rangle=\langle\mathscr{B}(X, Y), N\rangle$. Finally, the mean curvature of the immersion $\phi$ is $H=1 / 2 \operatorname{trace}\left(A_{N}\right)$.

Definition 2.1. A variation of $\phi$ is a smooth map $F:(-\epsilon, \epsilon) \times \Sigma \rightarrow \mathbb{R}_{+}^{3}$ such that for all $t \in(-\epsilon, \epsilon)$ the map $\phi_{t}: \Sigma \rightarrow \mathbb{R}_{+}^{3}, p \mapsto F(t, p)$ is an immersion and satisfies $\phi_{0}=\phi$.

For $p \in \Sigma$, we define the variation vector field of $F$ by $X(p)=\partial \phi_{t}(p) /\left.\partial t\right|_{t=0}$ and the normal variation function of $F$ by $f(p)=\langle X(p), N(p)\rangle$. We say that the variation $F$ is normal if $X$ is normal to $\phi$ at each point; we say $F$ has compact support if $X$ has compact support. For a variation with compact support and for small values of $t$, we have that $\phi_{t}$ is an immersion of $\Sigma$ in $\mathbb{R}_{+}^{3}$. In this case the area function $A:(-\epsilon, \epsilon) \rightarrow \mathbb{R}$ is given by

$$
A(t)=\int_{\Sigma} d A_{t}=\int_{\Sigma} \sqrt{\operatorname{det}\left(\left(d \phi_{t}\right)^{*}\left(d \phi_{t}\right)\right)} d A,
$$

where $d A$ is the area element of $\Sigma$. The function $A(t)$ is the area of $\Sigma$ with the metric induced by $\phi_{t}$. We also define the volume function $V:(-\epsilon, \epsilon) \rightarrow \mathbb{R}$ by

$$
V(t)=-\int_{[0, t] \times \Sigma} F^{*} d\left(\mathbb{R}_{+}^{3}\right),
$$

where $d\left(\mathbb{R}_{+}^{3}\right)$ is the volume element of $\mathbb{R}_{+}^{3}$ and $F^{*} d\left(\mathbb{R}_{+}^{3}\right)$ is the pull-back of $d\left(\mathbb{R}_{+}^{3}\right)$ by $F$. The function $V(t)$ does not actually represent the volume of some region with $\phi_{t}(\Sigma)$ as boundary, but of a "tubular neighborhood" along $\phi(\Sigma)$ between $\phi(\Sigma)$ and $\phi_{t}(\Sigma)$. The sign is related to the net change with respect to the normal field defining the orientation; for example, contracting a sphere in $\mathbb{R}^{3}$, which means moving it in the direction of the mean curvature vector, gives the expected negative sign for $V(t)$.

Definition 2.2. Let $F:(-\epsilon, \epsilon) \times \Sigma \rightarrow \mathbb{R}_{+}^{3}$ be a variation of $\phi$. We say $F$ preserves volume if $V(t)=V(0)$ (which is equal to zero) for all $t \in(-\epsilon, \epsilon)$. We say $F$ is admissible if $F(\partial \Sigma) \subset \Pi$ for all $t \in(-\epsilon, \epsilon)$.

Definition 2.3. We say that the immersion $\phi$ is stationary if $A^{\prime}(0)=0$ for all admissible variations that preserve volume.

Remark 2.4. Suppose $\Omega$ is a (compact) regular region in the slab $\mathscr{F}$ between the horospheres $\Pi$. Then by taking $\Sigma$ in Definition 2.2 as the (embedded regular) free boundary of $\Omega$, we may extend the variational approach above to produce a variation $\Omega(t)$ of $\Omega$ by embedded domains (for small $t$ ), such that the condition $V(t)=0$ in Definition 2.2 is equivalent to holding $|\Omega(t)|$ equal to $\Omega(0)$ along 
the variation. This justifies saying that the variation "preserves volume" in the definition above.

We now restate our problem. Let $\Pi_{1}$ and $\Pi_{2}$ be two parallel horospheres in $\mathbb{R}^{3}$, and let $\mathscr{F}=\mathscr{F}\left(\Pi_{2}, \Pi_{2}\right)$ be the (closed) slab between them.

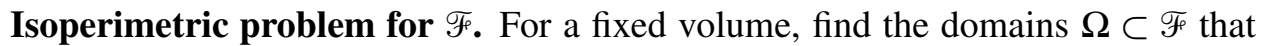
have minimal free boundary area.

Definition 2.5. A (compact) minimizing region $\Omega$ for this problem will be called an isoperimetric domain or region in $\mathscr{F}$.

More precisely, one looks to classify and describe geometrically the isoperimetric regions (as a function of the volume), that is, to find the isoperimetric profile (minimal free boundary area as a function of the prescribed volume) for $\mathscr{F}$.

\section{First results about the isoperimetric solutions}

Here, we characterize the stationary immersions according to Definition 2.3. The formulas below for the first variations of the area and volume functions are well known. For an immersed surface with boundary, the exterior conormal is the vector field along the boundary given as follows: In the tangent plane of $\Sigma$ at $p \in \partial \Sigma=\Gamma$, take the outward unitary vector orthogonal to the tangent vector to $\Gamma$ at $p$.

Proposition 3.1. Let $F$ be a variation of $\phi$ with variational field $X$ and compact support in $\Sigma$. Then

(1) $A^{\prime}(0)=-2 \int_{\Sigma} H f d A+\int_{\Gamma}\langle X, v\rangle d \Gamma$, where $v$ is the unitary exterior conormal, $d A$ is the element of area of $\Sigma$ and $d \Gamma$ is the element of length of $\Gamma$ induced by $\phi$;

(2) $V^{\prime}(0)=-\int_{\Sigma} f d A$, where $f(p)=\langle X(p), N(p)\rangle$.

Proof. Although the formula of the variation of the area functional is well known (see [Barbosa et al. 1988]), here we show a different way to deduce it. From the definition of $A(t)$, we obtain

$$
\begin{aligned}
A^{\prime}(t)=\int_{\Sigma}\left(\frac{1}{2 \sqrt{\operatorname{det}\left(\left(d \phi_{t}\right)^{*} d \phi_{t}\right)}} \operatorname{det}\right. & \left.\left(d \phi_{t}\right)^{*} d \phi_{t}\right) \\
\times & \left.\operatorname{trace}\left(\left(\left(d \phi_{t}\right)^{*} d \phi_{t}\right)^{-1} \circ \frac{d}{d t}\left(\left(d \phi_{t}\right)^{*} d \phi_{t}\right)\right)\right) d A .
\end{aligned}
$$

Since $\phi_{0}$ is the inclusion of $\Sigma$ in $\mathbb{R}_{+}^{3}, d \phi_{0}$ is the inclusion of the respective tangent spaces and $d \phi_{0}^{*}$ is the orthogonal projection on $T \Sigma$.

By evaluating $A^{\prime}(t)$ for $t=0$, we get

$$
A^{\prime}(0)=\int_{\Sigma} \frac{1}{2} \operatorname{trace}\left(\left.\frac{d}{d t}\right|_{t=0}\left(\left(d \phi_{t}\right)^{*} d \phi_{t}\right)\right) d A
$$


We apply the symmetry lemma for $\nabla^{\phi}$ along the immersion and get

$$
\left.\frac{d}{d t}\right|_{t=0}\left(d \phi_{t}\right)=\left.\nabla^{\phi} \frac{\partial \phi_{t}}{\partial t}\right|_{t=0}=\nabla^{\phi} X \text {. }
$$

Then

$$
A^{\prime}(0)=\int_{\Sigma} \frac{1}{2} \operatorname{trace}\left(\left.\left(\nabla^{\phi} X\right)^{*}\right|_{T \Sigma}+\operatorname{proj}_{T \Sigma} \nabla^{\phi} X\right) d A=\int_{\Sigma} \operatorname{trace}\left(\operatorname{proj}_{T \Sigma} \nabla^{\phi} X\right) d A
$$

where $\operatorname{proj}_{T \Sigma}$ denotes the projection on $T \Sigma$.

By decomposing the variational field as $X=X^{T}+X^{N}$, the projections of the tangent and normal components of $\nabla^{\phi}(X)$ on $T \Sigma$ are

$$
\operatorname{proj}_{T \Sigma} \nabla^{\phi}\left(X^{T}\right)=\nabla\left(X^{T}\right) \text { and } \operatorname{proj}_{T \Sigma} \nabla^{\phi}\left(X^{N}\right)=-\mathscr{A}_{X^{N}}
$$

where $\mathscr{A}_{X^{N}}$ is the Weingarten operator on $\Sigma$. Therefore

$$
A^{\prime}(0)=\int_{\Sigma}\left(\operatorname{div} X^{T}-2\left\langle X^{N}, H N\right\rangle\right) d A .
$$

We apply Stokes's theorem and get

$$
A^{\prime}(0)=\int_{\Gamma}\left\langle X^{T}, v\right\rangle d \Gamma-2 \int_{\Sigma}\left\langle X^{N}, H N\right\rangle d A=-2 \int_{\Sigma} H f d A+\int_{\Gamma}\langle X, v\rangle d \Gamma .
$$

The first variation of volume given in (2) is standard and its proof will be omitted; see [Barbosa et al. 1988].

From the next result we conclude that the boundary of our isoperimetric region must be an $H$-surface that contacts the horospheres $\Pi_{1}$ and $\Pi_{2}$ perpendicularly.

Theorem 3.2. Let $\phi: \Sigma \rightarrow \mathbb{R}_{+}^{3}$ be an immersion with boundary $\Gamma=\partial \Sigma$. Let $\Pi=\Pi_{1} \cup \Pi_{2}$ be the horospheres containing $\Gamma$. Then $\phi$ is stationary if and only if it has constant mean curvature and intersects $\Pi$ (if it does) perpendicularly along $\Gamma$.

Proof. We may show the reverse implication by adapting the proof of [Barbosa and do Carmo 1984, Proposition 2.7]. To show that $\phi$ meets $\Pi$ perpendicularly along $\Gamma$ if $\phi$ is stationary, we take an admissible variation $\Phi$ that preserves volume with variational field $X$, and we take $p_{0} \in \partial \Sigma$. Suppose by contradiction that $\left\langle X\left(p_{0}\right), v\left(p_{0}\right)\right\rangle \neq 0$. By continuity there is a neighborhood $U=W_{1} \cap \partial \Sigma$ of $p_{0}$ such that $\langle X(p), v(p)\rangle>0$ for all $p \in U$, where $W_{1}$ is a neighborhood of $p_{0}$ in $\Sigma$. Take $q \in \stackrel{\circ}{\Sigma} \backslash W_{1}$, let $W_{2}$ be a neighborhood of $q$ disjoint from $W_{1}$, and let $\mathscr{P}$ be a partition of unity on $W_{1} \bigcup W_{2}$. There exists a differentiable function $\xi_{1}: W_{1} \rightarrow \mathbb{R}$ such that $\xi_{1}\left(W_{1}\right) \subset[0,1]$ and with support $\operatorname{supp} \xi_{1} \subset W_{1}$. We may also take a differentiable map $\xi_{2}: W_{2} \rightarrow \mathbb{R}$ such that $\xi_{2}\left(W_{2}\right) \subset[0,1]$, supp $\xi_{2} \subset W_{2}$ and

$$
\int_{W_{1}} \xi_{1} f d W_{1}+\int_{W_{2}} \xi_{2} f d W_{2}=0
$$


Define a variation $\Phi_{\xi}:(-\epsilon, \epsilon) \times \Sigma \rightarrow \mathbb{R}$ with compact support on $W_{1} \bigcup W_{2}$ by

$$
\Phi_{\xi}^{t}(p)=\Phi_{\xi}(t, p)= \begin{cases}\Phi\left(\xi_{1} t, p\right) & \text { if } p \in W_{1} \\ \Phi\left(\xi_{2} t, p\right) & \text { if } p \in W_{2}\end{cases}
$$

Note that $\Phi_{\xi}$ is admissible because $\Phi$ is admissible.

If $f_{\xi}(p)$ denotes the normal component of the variation vector, we have

$$
\int_{\Sigma} f_{\xi}(p) d A=\int_{W_{1}} \xi_{1}(p) f(p) d W_{1}+\int_{W_{2}} \xi_{2}(p) f(p) d W_{2}=0,
$$

and $\Phi_{\xi}$ preserves volume.

For this variation we have

$$
0=A^{\prime}(0)=-2 H \int_{\Sigma} f_{\xi} d A+\int_{W_{1}} \xi_{1}\langle X, v\rangle d \Gamma=\int_{W_{1}} \xi_{1}\langle X, v\rangle d \Gamma>0,
$$

which is a contradiction. Then for all $p \in \partial \Sigma$, it follows that $\langle X, v\rangle(p)=0$.

Next we show that the isoperimetric domains are rotationally invariant.

We need some symmetrization principle for $H$-surfaces. By taking the hyperbolic version of Aleksandrov's principle of reflection (for further references and details, see [Aleksandrov 1962]) and using [Barbosa and Sa Earp 1998] to specialize to the case of reflection planes, we get the next result. A detailed proof may be found in [López 2006].

Theorem 3.3. Suppose $\Sigma$ is a compact, connected, orientable and embedded $H$ surface of class $C^{2}$ that lies between two parallel horospheres $\Pi_{1}$ and $\Pi_{2}$ in $\mathbb{R}_{+}^{3}$ and has boundary $\partial \Sigma \subset \Pi_{1} \cup \Pi_{2}$ (possibly empty). Then $\Sigma$ is rotationally symmetric around an axis perpendicular to $\Pi_{1}$ and $\Pi_{2}$.

We observe that the intersection of $\Sigma$ with a horosphere $\mathscr{H}$ (represented by a horizontal Euclidean plane) is just a Euclidean circle. In fact, if there were two concentric circles and the isoperimetric region $\mathscr{R}$ was delimited by these circles, we would apply the Aleksandrov's reflection principle with respect to vertical Euclidean planes and get a totally geodesic symmetry plane $P$ determined by the first contact point $x_{0}$; see Figure 2 . However, $P$ would obviously not contain the axis of symmetry of $\mathscr{R}$. See [López 2006] for a detailed proof of this fact.

\section{Isoperimetric regions between horospheres in $\mathbb{R}_{+}^{3}$}

We now classify the rotational $H$-surfaces of $\mathbb{R}_{+}^{3}$ that lie between two parallel horospheres, have boundary contained in the horospheres, and intersect the horospheres perpendicularly. In so doing, we get all possible solutions for the isoperimetric problem in hyperbolic space, since the solutions must be regions delimited by these $H$-surfaces. We start with important results from the thesis of Barrientos [1995]. 


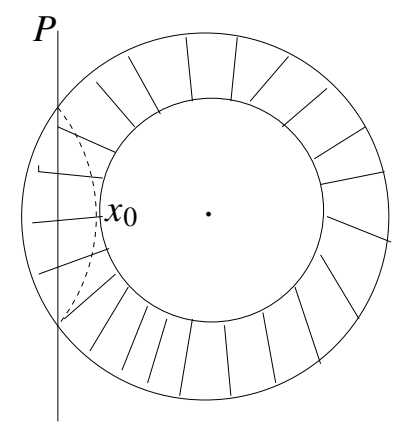

Figure 2. Excluded case: region between two concentric circles

If $(\rho, \theta, z)$ are the cylindrical coordinates of a point $p$ in $\mathbb{R}_{+}^{3}$, then the Cartesian coordinates are given by

$$
(\bar{x}, \bar{y}, \bar{z})=e^{z}(\tanh \rho \cos \theta, \tanh \rho \sin \theta, \operatorname{sech} \rho) .
$$

For a spherical rotational surface $\Sigma$ of $\mathbb{R}_{+}^{3}$ around the $\bar{z}$-axis, the orbit of a point $p=\left(\rho_{0}, \theta_{0}, z_{0}\right)$ is $\mathscr{R}_{\varphi}(p)=\left(\rho_{0}, \theta_{0}+\varphi, z_{0}\right)$, where $\mathscr{R}_{\varphi}(p)$ denotes the rotation of $p$ with angle $\varphi$ around $\bar{z}$. If $c(s)$ is the profile curve of $\Sigma$ parametrized by arclength, we can parametrize $\Sigma$ as $X(s, t)=\mathscr{R}_{t}(c(s))$, so that its metric is $d \sigma^{2}=$ $d s^{2}+U^{2}(s) d t^{2}$, where $U=U(s)$ is a positive function, $s$ is the arclength parameter of $c(s)$, and $d t=d \varphi$. We call $(s, t)$ the natural parameters of $\Sigma$. From (4-1), the metric (2-1) is given by

$$
d \rho^{2}+\sinh ^{2} \rho d \theta^{2}+\cosh ^{2} \rho d z^{2} .
$$

In the plane $\theta=0$, the profile curve $c(s)$ can be locally viewed as the graph $z=\lambda(s)=\lambda(\rho(s))$. From (4-2) we have in this parameterization that $d t=d \varphi$, $d s=\left(1+\lambda^{\prime 2}(\rho) \cosh ^{2} \rho\right)^{1 / 2} d \rho$,

$$
U^{2}(s)=\sinh ^{2} \rho(s) \quad \text { and } \quad \lambda^{\prime 2}(s)=\frac{1+U^{2}(s)-U^{\prime 2}(s)}{\left(1+U^{2}(s)\right)^{2}} .
$$

Then the natural parametrization for a rotational surface in cylindrical coordinates is

$$
\sinh ^{2} \rho(s)=U^{2}(s), \quad \lambda(s)=\int_{0}^{s} \frac{\sqrt{1+U^{2}(t)-U^{\prime 2}(t)}}{1+U^{2}(t)} d t, \quad \varphi(t)=t .
$$

Barrientos [1995] classified the $H$-surfaces of $\mathbb{R}_{+}^{3}$; another important reference is [Sterling 1987]. By setting $\zeta(s)=U^{2}(s)$, Barrientos found that the differential equation for the rotational $H$-surfaces in $\mathbb{R}_{+}^{3}$ is

$$
\zeta^{\prime 2} / 4=\left(1-H^{2}\right) \zeta^{2}+(1+2 a H) \zeta-a^{2},
$$


and showed that the behavior of their profile curves is determined by the constant of integration $a$. After choosing the surface orientation so that $H \geq 0$, there are three cases to study: $H=1, H \in[0,1)$ and $H>1$.

Next we give in each of these cases the natural parametrizations for a rotational $H$-surface in $\mathbb{R}_{+}^{3}$ generated by a curve $c(s)=(\rho(s), \lambda(s))$.

$H=1 \quad\left\{\begin{array}{l}\sinh ^{2} \rho(s)=\frac{a^{2}+(1+2 a)^{2} s^{2}}{1+2 a}, \\ \lambda(s)=\int_{0}^{s} \frac{\sqrt{1+2 a}\left(-a(1+a)+(1+2 a)^{2} t^{2}\right) \sqrt{a^{2}+(1+2 a)^{2} t^{2}}}{\left(-a(1+a)+(1+2 a)^{2} t^{2}\right)^{2}+(1+2 a)^{4} t^{2}} d t, \\ \varphi(t)=t .\end{array}\right.$
$H \in[0,1)\left\{\begin{array}{l}\sinh ^{2} \rho(s)=\frac{-A+B \cosh (2 \alpha s)}{2 \alpha^{2}}, \\ \lambda(s)=\int_{0}^{s} \frac{\sqrt{2} \alpha(-2 a+H(-1+B \cosh (2 \alpha t))) \sqrt{-A+B \cosh (2 \alpha t)}}{(-2 a+H(-1+B \cosh (2 \alpha t)))^{2}+\alpha^{2} B^{2} \sinh ^{2}(2 \alpha t)} d t, \\ \varphi(t)=t,\end{array}\right.$

where $A=1+2 a H, B=\sqrt{1+4 a H+4 a^{2}}$ and $\alpha=\sqrt{1-H^{2}}$.

$H>1\left\{\begin{array}{l}\sinh ^{2} \rho(s)=\frac{A+B \sin (2 \alpha s)}{2 \alpha^{2}}, \\ \lambda(s)=\int_{0}^{s} \frac{\sqrt{2} \alpha(2 a+H(1+B \sin (2 \alpha t))) \sqrt{A+B \sin (2 \alpha t)}}{(2 a+H(1+B \sin (2 \alpha t)))^{2}+\alpha^{2} B^{2} \cos ^{2}(2 \alpha t)} d t, \\ \varphi(t)=t,\end{array}\right.$

where $A=1+2 a H, B=\sqrt{1+4 a H+4 a^{2}}$ and $\alpha=\sqrt{H^{2}-1}$.

Now we introduce some notations and definitions used throughout this section. From (4-1) the profile curve of a rotational $H$-surface in $\mathbb{R}_{+}^{3}$ is given by

$$
c_{+}(s)=e^{\lambda(s)}(\tanh \rho(s), \operatorname{sech} \rho(s)) .
$$

Here $\rho(s)$ and $\lambda(s)$ are determined by the suitable parametrization above. In the $H=1$ case, $a>-1 / 2$. When $-1 / 2<a<0$, we say the rotational surfaces are of catenoid cousin type. In the $H \in[0,1)$ case, $a \in \mathbb{R}$. When $a<0$, we say the rotational surfaces are of equidistant type. In the $H>1$ case, we have $a \geq\left(-H+\sqrt{H^{2}-1}\right) / 2$. When $-1 /(4 H)<a<0$, we obtain the onduloid type surfaces. In each case, we get umbilical surfaces when $a=0$.

By taking $\lambda=0$ in (4-4), we get the curve $c_{g}(s)=(\tanh \rho(s)$, sech $\rho(s))$, which is an upper half-circle perpendicular to the $\bar{z}$-axis. Namely, it is a geodesic with Euclidean radius $r=\left(\tanh ^{2} \rho(s)+\operatorname{sech}^{2} \rho(s)\right)^{1 / 2}=1$. The curve $c_{g}(s)$ is called the geodesic radius. Our analysis works up to Euclidean homotheties $\mathscr{H}_{r}$ for general 
$r>0$, namely

$$
\mathscr{H}_{r}\left(c_{+}(s)\right)=e^{\lambda(s)}(r \tanh \rho(s), r \operatorname{sech} \rho(s)) ;
$$

these give other families of profile curves of rotational $H$-surfaces. For the results in this section that deal with geodesic radius, we take $r=1$. By Theorems 3.2 and 3.3, the boundaries of the isoperimetric solutions must be rotational $H$-surfaces that meet the horospheres $\left\{z=c_{1}\right\}$ and $\left\{z=c_{2}\right\}$ perpendicularly. Now our goal is to determine the vertical tangency points of the profile curves of the rotational surfaces.

Definition 4.1. Suppose $c_{+}(s)$ is a curve parametrized by (4-4). We say that a point $c_{+}(s)$ is a vertical tangency point if the tangent vector at $c_{+}(s)$ satisfies $c_{+}^{\prime}(s)=(0, b)$, where $b \in \mathbb{R}^{*}$, that is,

$$
\begin{aligned}
& e^{\lambda(s)}\left(\tanh \rho(s) \lambda^{\prime}(s)+\operatorname{sech}^{2} \rho(s) \rho^{\prime}(s)\right)=0, \\
& e^{\lambda(s)}\left(\operatorname{sech} \rho(s) \lambda^{\prime}(s)-\operatorname{sech} \rho(s) \tanh \rho(s) \rho^{\prime}(s)\right)=b .
\end{aligned}
$$

Since $e^{\lambda(s)}>0$, Equation (4-5) implies that

$$
\tanh \rho(s) \lambda^{\prime}(s)+\operatorname{sech}^{2} \rho(s) \rho^{\prime}(s)=0 .
$$

By (4-7) we obtain the points where the vertical tangency occurs, and by (4-6) we get the direction of the tangency (upward or downward).

By applying (4-3) to (4-7) we see that, if $p$ is a vertical tangency point with $U(s) \neq 0$, then

$$
U^{2}(s)=U^{\prime 2}(s)
$$

and the roots of (4-8) give us the vertical tangency points.

Next we study the behavior of the profile curve of rotational $H$-surfaces and determine the possible vertical tangency points. In each case, $\rho(s), \lambda(s)$ and $U(s)$ are those the of corresponding parametrization on page 11 .

\section{The case $H=1$.}

Theorem 4.2. If $c_{+}(s)=e^{\lambda(s)}(\tanh \rho(s), \operatorname{sech} \rho(s))$ is the parametrization of the profile curve of a rotational $H$-surface in $\mathbb{R}_{+}^{3}$ with $H=1$, then $c_{+}(s)$ is symmetric with respect to the geodesic radius $c_{g}$.

Proof. By definition, $\lambda(0)=0$, so $c_{+}(0) \in c_{g}$. If $I$ is the Euclidean inversion through $c_{g}$, then $I\left(c_{+}(s)\right)=c_{+}(-s)$, because $\rho(s)$ is even and $\lambda(s)$ is odd.

By the definition of $\rho(s)$,

$$
\sinh \rho(s)=0 \quad \text { if and only if } \quad a=0 \text { and } s=0 .
$$


So $\tanh \rho(s)>0$ if neither $a$ nor $s$ is zero. Furthermore $s=0$ is the unique minimum point of $\rho(s)$.

As for $U(s)$ in the case $H=1$, we have

$$
U^{2}(s)=\frac{a^{2}+(1+2 a)^{2} s^{2}}{1+2 a} \quad \text { and } \quad U^{\prime 2}(s)=\frac{(1+2 a)^{3} s^{2}}{a^{2}+(1+2 a)^{2} s^{2}} .
$$

By applying (4-10) to (4-8) it follows that

$$
(1+2 a)^{4} s^{4}+\left(2 a^{2}(1+2 a)^{2}-(1+2 a)^{4}\right) s^{2}+a^{4}=0 .
$$

This is a second order equation in $s^{2}$, with discriminant

$$
\Delta=(1+2 a)^{6}(4 a+1) .
$$

Since $1+2 a>0$, we have these facts in the $H=1$ case:

- If $-1 / 2<a<-1 / 4$, then (4-11) has no real roots, so there are no points of vertical tangency.

- If $a=-1 / 4$, there are at most two vertical tangency points

$$
s= \pm 1 / 2
$$

- If $a>-1 / 4$, there are at most four vertical tangency points, given by

$$
\begin{array}{ll}
s_{1}=\frac{1+2 a+\sqrt{1+4 a}}{2(1+2 a)}, & s_{2}=-s_{1}, \\
s_{3}=\frac{1+2 a-\sqrt{1+4 a}}{2(1+2 a)}, & s_{4}=-s_{3} .
\end{array}
$$

Besides these pieces of information, we study the vertical tangencies as the parameter $a$ varies.

The subcase $-1 / 4 \leq a<0$. In this case, we have $\lambda^{\prime}(s)>0$. If $s \geq 0$, then

$$
\tanh \rho(s) \lambda^{\prime}(s)+\operatorname{sech}^{2} \rho(s) \rho^{\prime}(s)>0,
$$

and (4-7) is not possible. Since $1+2 a>0$, the roots $s_{1}$ and $s_{3}$ of (4-8) given by (4-14) are strictly positive and thus do not give vertical tangency points. The other roots $s_{2}, s_{4}<0$ give us the vertical tangency points with upward direction for $b>0$ in (4-6). The left figure in Figure 3 shows the profile curve for $H=1$ and $a=-0.2$ and the horocycles that pass through the vertical tangency. To the right, we see two parallel horospheres and the rotational surface between the horospheres that meets them perpendicularly.

In particular, if $a=-1 / 4$, the positive root $s=1 / 2$ of (4-8) given by (4-13) does not give a vertical tangency point, and there is only one vertical tangency 

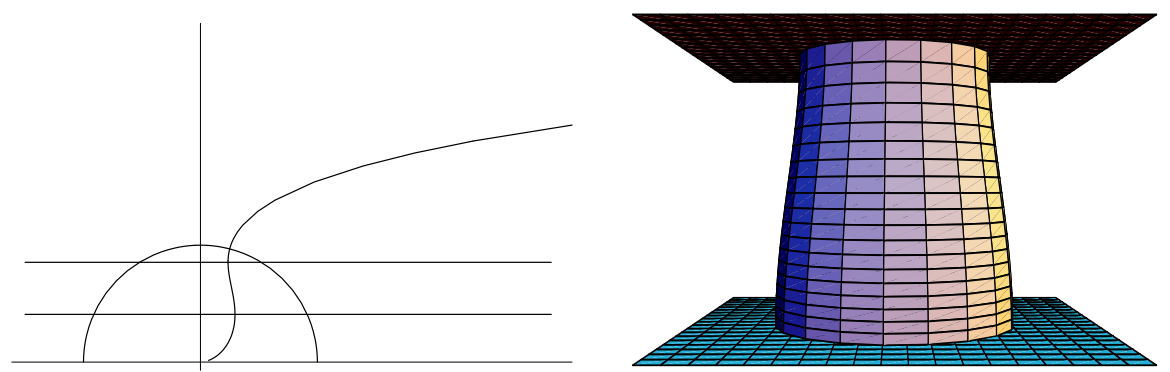

Figure 3. At left, the profile curve for $H=1$ and $a=-0.2$, and at right the corresponding rotational surface.
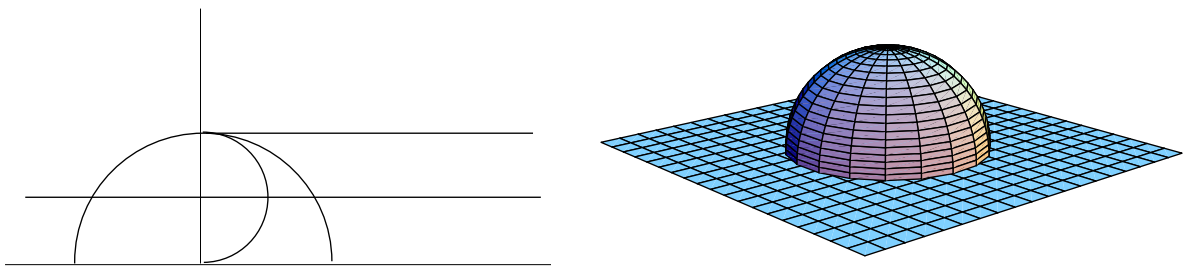

Figure 4. At left, the profile curve for $H=1$ and $a=0$, and at right the corresponding rotational surface.

point corresponding to $s=-1 / 2$. From the definitions of $\rho(s)$ and $\lambda(s)$ we get

$$
\begin{aligned}
& \lim _{s \rightarrow-\infty} e^{\lambda(s)} \tanh \rho(s)=0, \quad \lim _{s \rightarrow-\infty} e^{\lambda(s)} \operatorname{sech} \rho(s)=0, \\
& \lim _{s \rightarrow \infty} e^{\lambda(s)} \tanh \rho(s)=\infty, \quad \lim _{s \rightarrow-\infty} e^{\lambda(s)} \operatorname{sech} \rho(s)=\infty .
\end{aligned}
$$

Geometrically, one sees it's impossible to get an isoperimetric region in this case.

The subcase $a=0$. In this case, two pieces of horocycle tangent at $(0,1)$ generate the umbilical surfaces with $H=1$. They are represented by the Euclidean plane $\{z=1\}$ or the Euclidean sphere with radius $1 / 2$ and tangent to $\partial \mathbb{R}_{+}^{3}$ at $(0,0,0)$. In the latter case there is only one vertical tangency point and the surface boundary meets only one of the horospheres perpendicularly. In fact, by taking the upper Euclidean half-sphere that represents the horosphere, we get the possible isoperimetric solution for the umbilical case with $H=1$. Figure 4 illustrates the situation.

The subcase $a>0$. In this case, the profile curves have only one self-intersection. From (4-4), if $c_{+}\left(s_{i}\right)=c_{+}\left(s_{j}\right)$ is a self-intersection, then $s_{i}= \pm s_{j}$. Since the curves are symmetric with respect to $c_{g}$, the self-intersections must occur on $c_{g}$. So $\lambda\left(s_{i}\right)=\lambda\left(s_{j}\right)=0$. By its definition in this case, we deduce that $\lambda(s)$ has a maximum at $-\sqrt{a(1+a)} /(1+2 a)$ and a minimum at $\sqrt{a(1+a)} /(1+2 a)$. Furthermore, $\lim _{s \rightarrow \infty} \lambda(s)=\infty$ (see [Barrientos 1995]), $\lambda(0)=0$, and $\lambda(s)$ is an odd function. 

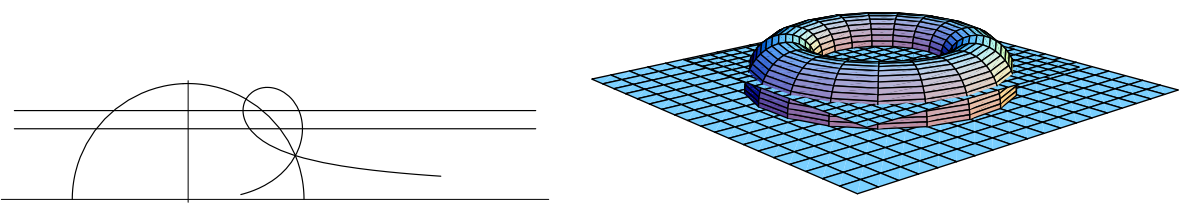

Figure 5. At left, the profile curve for $H=1$ and $a=1$, and at right the corresponding rotational surface. This case cannot be an isoperimetric solution.

Also, $\rho(s)$ has only one minimum at $s=0$. So, if $s>\sqrt{a(1+a)} /(1+2 a)>0$ then $\rho^{\prime}(s), \lambda^{\prime}(s)>0$ and

$$
\tanh \rho(s) \lambda^{\prime}(s)+\operatorname{sech}^{2} \rho(s) \rho^{\prime}(s)>0 .
$$

If however $-\sqrt{a(1+a)} /(1+2 a)<s<0$, then $\rho^{\prime}(s), \lambda^{\prime}(s)<0$, which implies that

$$
\tanh \rho(s) \lambda^{\prime}(s)+\operatorname{sech}^{2} \rho(s) \rho^{\prime}(s)<0 .
$$

In either case, (4-7) does not hold. Since $a>0$, the roots $s_{1}, s_{2}, s_{3}$ and $s_{4}$ of (4-8) given by (4-14) satisfy

$$
s_{2}<-\frac{\sqrt{a(1+a)}}{1+2 a}<s_{4}<0<s_{3}<\frac{\sqrt{a(1+a)}}{1+2 a}<s_{1},
$$

Therefore, vertical tangency is possible only for the positive roots $s_{2}$ and $s_{3}$. Since $\rho^{\prime}\left(s_{2}\right)<0$ and $\lambda^{\prime}\left(s_{2}\right)>0$, the vertical tangency for $s_{2}$ is upward. However, it is downward for $s_{3}$, since $\rho^{\prime}\left(s_{3}\right)>0$ and $\lambda^{\prime}\left(s_{3}\right)<0$. The isoperimetric solution is not possible in this case because, if the vertical tangencies did not occur at the same height, then a piece of the rotational surface would be outside of the region between the horospheres; see Figure 5.

Even if vertical tangency occurred at the same height, the intersection of the rotational $H$-surface with the parallel horospheres would be two concentric circles, which is not possible due to Theorem 3.3.

In summary, for $H=1$ the boundary of the region $\Omega$ must be either a catenoid cousin-type surface as in Figure 3 or umbilical as in Figure 4

We proceed in the analogous way to study the other cases, and give here only the main equations and results.

The case $H \in[0,1)$.

Theorem 4.3. If $c_{+}(s)=e^{\lambda(s)}(\tanh \rho(s), \operatorname{sech} \rho(s))$ is the parametrization of the profile curve of a rotational $H$-surface in $\mathbb{R}_{+}^{3}$ with $H \in[0,1)$, then

(1) $c_{+}(s)$ is symmetric with respect to the geodesic radius $c_{g}$, and 
(2) the asymptotic boundary of the profile curves consists of one or two points.

Proof. The proof of (1) is similar to that of Theorem 4.2. Barrientos [1995] showed that $\rho(s)$ is unbounded but $\lambda(s)$ is bounded and has a limit. Then we have $\lim _{|s| \rightarrow \infty} e^{\lambda(s)} \operatorname{sech} \rho(s)=0$, and the asymptotic boundary of the profile curves consists of one or two points.

Since $\sinh \rho(s) \geq 0$, we have by definition (page 11) that $\sinh \rho(s)=0$ if and only if $a=0$ and $s=0$.

Again by definition, it follows that

$$
U^{2}(s)=\frac{-A+B \cosh (2 \alpha s)}{2 \alpha^{2}} \quad \text { and } \quad U^{\prime 2}(s)=\frac{B^{2} \sinh ^{2}(2 \alpha s)}{2(-A+B \cosh (2 \alpha s))}
$$

By applying (4-16) to (4-8), we get

$$
B^{2} H^{2} \cosh ^{2}(2 \alpha s)-2 A B \cosh (2 \alpha s)+A^{2}+\alpha^{2} B^{2}=0 .
$$

This is a second order equation in $\cosh (2 \alpha s)$, with discriminant

$$
\Delta=4 B^{2}\left(1-H^{2}\right)^{2}(1+4 a H) .
$$

Since $B>0$ if $H \in(0,1)$ and $a$ is defined for any real, we have these facts:

- If $a<-1 /(4 H)$, there are no vertical tangency points.

- If $a=-1 /(4 H)$, there are at most two vertical tangency points

$$
s= \pm \frac{1}{2 \alpha} \operatorname{arccosh}(1 / H) \text {. }
$$

- If $a>-1 /(4 H)$, there are at most four vertical tangency points

$$
\begin{array}{ll}
s_{1}=\frac{1}{2 \alpha} \operatorname{arccosh}\left(\frac{A+\left(1-H^{2}\right) \sqrt{1+4 a H}}{B H^{2}}\right), & s_{2}=-s_{1}, \\
s_{3}=\frac{1}{2 \alpha} \operatorname{arccosh}\left(\frac{A-\left(1-H^{2}\right) \sqrt{1+4 a H}}{B H^{2}}\right), & s_{4}=-s_{3} .
\end{array}
$$

In particular, $H=0$ in (4-17) gives $2 B \cosh (2 s)-1-B^{2}=0$, whose solutions are $s= \pm(1 / 2) \operatorname{arccosh}\left(B^{2}+1 /(2 B)\right)$.

First, let us specialize to the case that $H \in(0,1)$; we'll treat $H=0$ later.

The subcase $-1 /(4 H) \leq a<0$. In this case, only the roots $s_{2}, s_{4}<0$ give us vertical tangency points with upward direction. Figure 6 shows the profile curve for $H=0.5$ and $a=-0.25$ and the horocycles that pass through the vertical tangencies. The mean curvature vector for the part of the rotational surface in the interior of the totally geodesic (symmetry plane of the surface) points out toward the rotation axis, and so determines the isoperimetric region illustrated at right. 

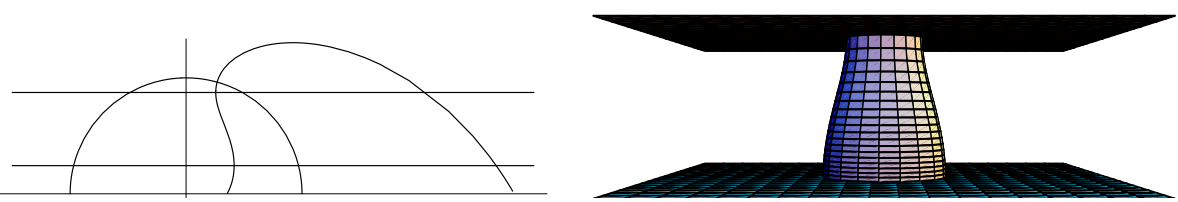

Figure 6. At left, profile curve for $H=0.5$ and $a=-0.25$, and at right the corresponding rotational surface.

In particular, if $a=-1 /(4 H)$ there is only one vertical tangency point at $s=$ $-1 /(2 \alpha) \operatorname{arccosh}(1 / H)<0$. Although the profile curve intersects the horocycle at another point, it is not of vertical tangency.

The subcase $a=0$. In this case, two pieces of equidistant curve tangent at $(0,1)$ generate the umbilical surfaces with $H \in(0,1)$. They are represented by pieces of Euclidean spheres tangent at $(0,0,1)$. The vertical tangency occurs only for the equidistant profile curve that is the nearest to the rotation axis. Since the mean curvature vector of this umbilical surface points outward to the rotation axis, it determines an isoperimetric region.

The subcase $a>0$. If $a>0$ only the roots $s_{2}$ and $s_{3}$ correspond to vertical tangencies with directions upward in $s_{2}$ and downward in $s_{3}$. Geometrically, one sees that it is impossible to get an isoperimetric region in this case.

As for the $H=0$ case, for $a<0$ or $a>0$ we get only one vertical tangency point. If $a=0$, the rotational surface is a totally geodesic plane. Thus it is not possible to get an isoperimetric region for any $a \in \mathbb{R}$.

Finally, we conclude that for $H \in[0,1)$ the boundary of the region $\Omega$ must be an equidistant-type surface (see Figure 6) or an umbilical surface with $H \in(0,1)$.

\section{The case $\mathrm{H}>1$.}

Theorem 4.4. If $c_{+}(s)=e^{\lambda(s)}(\tanh \rho(s)$, sech $\rho(s))$ is the parametrization of the profile curve of a rotational $H$-surface in $\mathbb{R}_{+}^{3}$ with $H>1$, then $c_{+}(s)$ is a periodic curve with period $\pi / \alpha$.

Proof. We show that the hyperbolic length of the segment with extremes $c_{+}(s)$ and $c_{+}(s+\pi / \alpha)$ is constant for all $s$. Barrientos [1995] shows that

$$
\rho(s+\pi / \alpha)=\rho(s) \quad \text { and } \quad \lambda(s+\pi / \alpha)=\lambda(s)+\lambda(\pi / \alpha),
$$

which implies from (4-4) that $c_{+}(s+\pi / \alpha)=e^{\lambda(\pi / \alpha)} c_{+}(s)$.

We fix $s_{0}$ and parametrize the segment with extremes $c_{+}\left(s_{0}\right)$ and $c_{+}\left(s_{0}+\pi / \alpha\right)$ by

$$
\beta(t)=\left(t, \frac{t}{\sinh \rho\left(s_{0}\right)}\right), \quad \text { with } e^{\lambda\left(s_{0}\right)} \tanh \rho\left(s_{0}\right) \leq t \leq e^{\lambda\left(s_{0}\right)} e^{\lambda(\pi / \alpha)} \tanh \rho\left(s_{0}\right) .
$$


Therefore its hyperbolic length is $L(\beta(t))=\lambda(\pi / \alpha) \cosh \rho\left(s_{0}\right)$.

The length of the segment depends only on the function $\rho(s)$ with period $\pi / \alpha$, given in (4-20). So $L(\beta(t))$ is the same for any $s_{0}$.

Again by definition, it follows that

$$
U^{2}(s)=\frac{A+B \sin (2 \alpha s)}{2 \alpha^{2}} \quad \text { and } \quad U^{\prime 2}(s)=\frac{B^{2} \cos ^{2}(2 \alpha s)}{2(A+B \sin (2 \alpha s))} .
$$

By applying (4-21) to (4-8), we get

$$
B^{2} H^{2} \sin ^{2}(2 \alpha s)+2 A B \sin (2 \alpha s)+A^{2}-\alpha^{2} B^{2}=0 .
$$

This is a second order equation in $\sin (2 \alpha s)$, with discriminant

$$
\Delta=4 B^{2}\left(H^{2}-1\right)^{2}(1+4 a H) .
$$

Since $H>1$ and $B>0$, this discriminant leads to these conclusions:

- If $a<-1 /(4 H)$, there are no vertical tangency points.

- If $a=-1 /(4 H)$, the possible vertical tangency points are ${ }^{1}$

$$
\begin{array}{ll}
s_{k}=\frac{1}{2 \alpha} \arcsin (1 / H)+k \pi / \alpha, & \text { for } k \in \mathbb{Z}, \\
\tilde{s}_{k}=\frac{1}{2 \alpha} \widehat{\arcsin }(1 / H)+k \pi / \alpha & \text { for } k \in \mathbb{Z} .
\end{array}
$$

- If $a>-1 /(4 H)$ the possibilities are, for $k \in \mathbb{Z}$,

$$
\begin{aligned}
& S_{k}=\frac{1}{2 \alpha} \arcsin \left(\mathscr{D}_{+}\right)+k \pi / \alpha, \quad s_{k}=\frac{1}{2 \alpha} \arcsin \left(\mathscr{D}_{-}\right)+k \pi / \alpha, \\
& \tilde{S}_{k}=\frac{1}{2 \alpha} \widehat{\arcsin }\left(\mathscr{D}_{+}\right)+k \pi / \alpha, \quad \tilde{s}_{k}=\frac{1}{2 \alpha} \widehat{\arcsin }\left(\mathscr{D}_{-}\right)+k \pi / \alpha,
\end{aligned}
$$

where $\mathscr{D}_{ \pm}=\left(-A \pm\left(H^{2}-1\right) \sqrt{1+4 a H}\right) /\left(B H^{2}\right)$

Now we determine when the vertical tangency really occurs, depending on the geometry of the profile curve.

(1) If $-1 /(4 H) \leq a<0$, only the roots $\tilde{S}_{k}$ and $\tilde{s}_{k}$ give the vertical tangency points with upward direction; see Figure 7.

(2) If $a=0$, we have tangent geodesic half-circles along the rotation axis, each of which generates a geodesic sphere in $\mathbb{R}_{+}^{3}$; these are umbilical surfaces and isoperimetric regions.

(3) If $a>0$, we may analyze the behavior of the profile curves in the interval ] $-\pi /(4 \alpha), 3 \pi /(4 \alpha)$, since by Theorem 4.4 they are $(\pi / \alpha)$-periodic. It is easy to see that only the roots $s_{0}$ and $\tilde{S}_{0}$ give vertical tangency with directions

\footnotetext{
${ }^{1}$ Here $\widetilde{\arcsin }$ is the inverse sine such that $\widetilde{\arcsin } x \in[\pi / 2,3 \pi / 2)$, and arcsin is the usual inverse.
} 


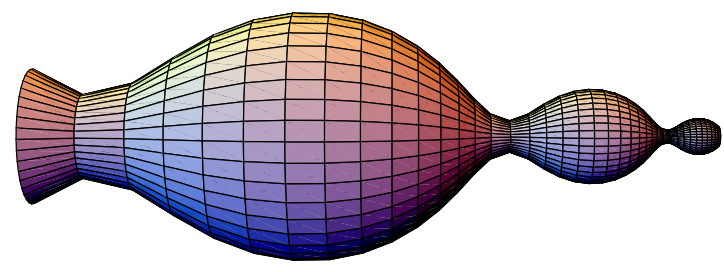

Figure 7. Hyperbolic onduloid with $H=3$ and $a=-0.05$.

downward in $s_{0}$ and upward in $\tilde{S}_{0}$. This case corresponds to the so-called hyperbolic nodoids, which are not embedded surfaces.

In summary, for $H>1$, the boundary of the region $\Omega$ can be either an onduloidtype surface as in Figure 7 or an umbilical surface.

Proof of Theorem 1.1. We start with the existence and then obtain the possible minimizing regions. By Theorem 3.3, the solutions to the isoperimetric problem have as boundaries rotationally invariant surfaces that have constant mean curvature where they are regular. But they must be regular (actually analytic), since the singularities along such boundaries must have, by well-known results, (Hausdorff) codimension at least 7, which is not possible for (2-dimensional) surfaces. Now, by results of [Morgan 1994], the existence of the isoperimetric solutions follows from the fact that $\mathscr{F}_{c_{1}, c_{2}} / G$ is compact, where $G$ is the group of isometries of $\mathbb{R}_{+}^{3}$ whose elements leave invariant the region $\mathscr{F}_{c_{1}, c_{2}}$ between the horospheres, that is, the rotations around a vertical geodesic and the horizontal translations. The second part of Theorem 1.1 follows from the analysis of vertical tangencies done in the cases $H=1, H \in[0,1)$, and $H>1$.

plus .5pt

\section{References}

[Aleksandrov 1962] A. D. Aleksandrov, "Uniqueness theorems for surfaces in the large, V", Amer. Math. Soc. Transl. (2) 21 (1962), 412-416. MR 27 \#698e Zbl 0119.16603

[Athanassenas 1987] M. Athanassenas, "A variational problem for constant mean curvature surfaces with free boundary", J. Reine Angew. Math. 377 (1987), 97-107. MR 88j:49027 Zbl 0604.53003 [Barbosa and do Carmo 1984] J. L. Barbosa and M. do Carmo, "Stability of hypersurfaces with constant mean curvature", Math. Z. 185:3 (1984), 339-353. MR 85k:58021c Zbl 0513.53002

[Barbosa and Sa Earp 1998] J. L. Barbosa and R. Sa Earp, "Prescribed mean curvature hypersurfaces in $H^{n+1}$ with convex planar boundary, II", pp. 43-79 in Séminaire de Théorie spectrale et géométrie, vol. 16, Institut Fourier, Université Grenoble I, 1998.

[Barbosa et al. 1988] J. L. Barbosa, M. do Carmo, and J. Eschenburg, "Stability of hypersurfaces of constant mean curvature in Riemannian manifolds", Math. Z. 197:1 (1988), 123-138. MR 88m:53109 Zbl 0653.53045

[Barrientos 1995] J. Barrientos, Superfícies helicoidais com curvatura média constante em espaços forma tridimensionais, thesis, Pontifícia Universidade Católica do Rio de Janeiro, 1995. 
[Finn 1986] R. Finn, Equilibrium capillary surfaces, Grundlehren der Mathematischen Wissenschaften 284, Springer, New York, 1986. MR 88f:49001 Zbl 0583.35002

[López 1999] R. López, "Constant mean curvature surfaces with boundary in hyperbolic space", Monatsh. Math. 127:2 (1999), 155-169. MR 2000m:53017 Zbl 0981.53053

[López 2006] R. López, "Symmetry of stationary hypersurfaces in hyperbolic space", Geom. Dedicata 119 (2006), 35-47. MR 2007m:58019 Zbl 1116.53008

[Morgan 1994] F. Morgan, "Clusters minimizing area plus length of singular curves", Math. Ann. 299:4 (1994), 697-714. MR 95g:49083 Zbl 0805.49025

[Morgan 2009] F. Morgan, Geometric measure theory: A beginner's guide, 4th ed., Academic Press, San Diego, 2009. MR 2009i:49001 Zbl 05501365

[Pedrosa and Ritoré 1999] R. H. L. Pedrosa and M. Ritoré, "Isoperimetric domains in the Riemannian product of a circle with a simply connected space form and applications to free boundary problems", Indiana Univ. Math. J. 48:4 (1999), 1357-1394. MR 2001k:53120

[Ritoré and Ros 2002] M. Ritoré and A. Ros, "Some updates on isoperimetric problems", Math. Intelligencer 24:3 (2002), 9-14. MR 2003g:53014

[Sterling 1987] I. Sterling, "A generalization of a theorem of Delaunay to rotational $W$-hypersurfaces of $\sigma_{l}$-type in $H^{n+1}$ and $S^{n+1}$, Pacific J. Math. 127:1 (1987), 187-197. MR 88c:53015 Zbl 0579. 53041

[Vogel 1987] T. I. Vogel, "Stability of a liquid drop trapped between two parallel planes", SIAM J. Appl. Math. 47:3 (1987), 516-525. MR 88e:53010 Zbl 0627.53004

Received October 24, 2008. Revised July 23, 2009.

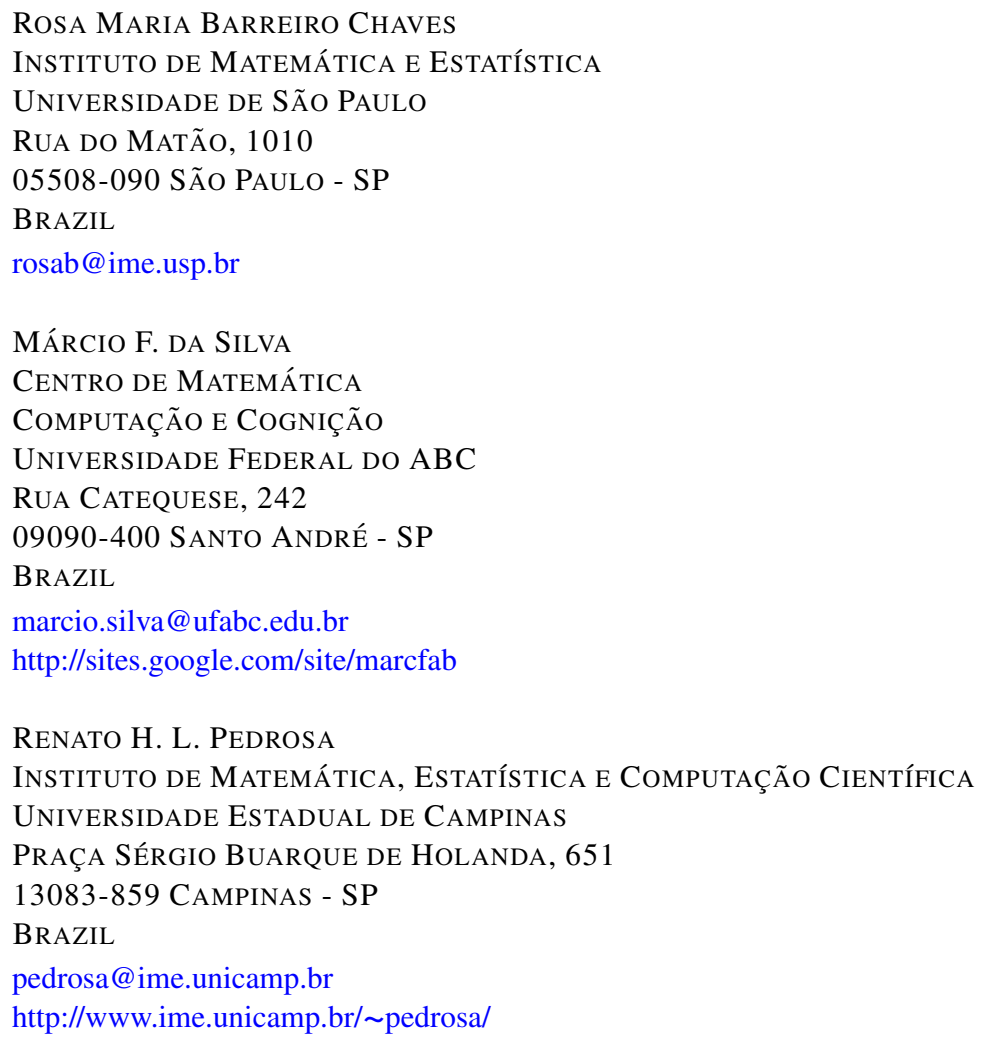

Renato H. L. Pedrosa

Instituto de MATEMÁtica, Estatística e Computação Científica

UNIVERSIDADE ESTADUAL DE CAMPINAS

PRAÇA SÉRgIO BuARQUE DE Holanda, 651

13083-859 CAMPINAS - SP

BRAZIL

pedrosa@ime.unicamp.br

http://www.ime.unicamp.br/ pedrosa/ 